Article

\title{
Electromagnet Weight Reduction in a Magnetic Levitation System for Contactless Delivery Applications
}

\section{Do-Kwan Hong ${ }^{1, *}$, Byung-Chul Woo ${ }^{1}$, Dae-Hyun Koo ${ }^{1}$ and Ki-Chang Lee ${ }^{1,2}$}

1 Electric Motor Research Center, Korea Electrotechnology Research Institute, Boolmosangil 70, Changwon, 641-120, Korea; E-Mails: bcwoo@keri.re.kr (B.-C.W.); dhk371@keri.re.kr (D.-H.K.)

2 School of Mechanical Engineering, Pusan National University, Geumjeong-gu, Busan, 609-735, Korea; E-Mail: leekc@pusan.ac.kr (K.-C.L.)

* Author to whom correspondence should be addressed; E-Mail: dkhong@keri.re.kr; Tel.: +82-55-280-1395; Fax: +82-55-280-1547.

Received: 1 June 2010; in revised form: 2 July 2010 / Accepted: 7 July 2010 /

Published: 9 July 2010

\begin{abstract}
This paper presents an optimum design of a lightweight vehicle levitation electromagnet, which also provides a passive guide force in a magnetic levitation system for contactless delivery applications. The split alignment of C-shaped electromagnets about $\mathrm{C}$-shaped rails has a bad effect on the lateral deviation force, therefore, no-split positioning of electromagnets is better for lateral performance. This is verified by simulations and experiments. This paper presents a statistically optimized design with a high number of the design variables to reduce the weight of the electromagnet under the constraint of normal force using response surface methodology (RSM) and the kriging interpolation method. 2D and $3 \mathrm{D}$ magnetostatic analysis of the electromagnet are performed using ANSYS. The most effective design variables are extracted by a Pareto chart. The most desirable set is determined and the influence of each design variable on the objective function can be obtained. The generalized reduced gradient (GRG) algorithm is adopted in the kriging model. This paper's procedure is validated by a comparison between experimental and calculation results, which shows that the predicted performance of the electromagnet designed by RSM is in good agreement with the simulation results.
\end{abstract}

Keywords: optimum design; response surface methodology (RSM); levitation control; kriging interpolation method; design of experiments (DOE); electromagnet 


\section{Introduction}

Electromagnetically levitated and guided systems with linear motor propulsion are commonly used in the field of people transport vehicles, tool machines and conveyor systems because of their silent and non-contact motion [1-3]. Passive guidance controls are normally used in real implementations of Maglev systems with linear induction motor propulsion to reduce construction costs and complexity. The Japanese HSST and the English BAMS (Birmingham Airport Maglev System) are well-known costeffective transport systems based on linear induction motor propulsion and magnetic levitation. In both systems, the guidance force needed to keep the vehicles on the track is obtained by the levitation electromagnets with the help of particular shapes of the rail and clever placement of the electromagnets about it. In Maglev transport systems where levitation magnets are on moving parts, the design of levitation electromagnet is the most important factor. It can account for a considerable part (10\%) of the whole vehicle weight, which can largely affect weight and stability of the magnetic levitation system. Optimum plans for the weight reduction of the levitation electromagnet are consequently desirable [4-7]. In this paper, an optimal design procedure was required in order to develop a magnetic levitation system for a contactless delivery application shown in Figure 1. As a first step, the design goal was to reduce the weight of the electromagnet of the magnetic levitation system with the constraint of normal force, considering the initial model. At a second step, the most effective design variables and their levels should be determined and be arranged in an orthogonal array table.

Figure 1. Magnetic levitation system prototype for contactless delivery application: (a) 3D modeling (b) prototype.

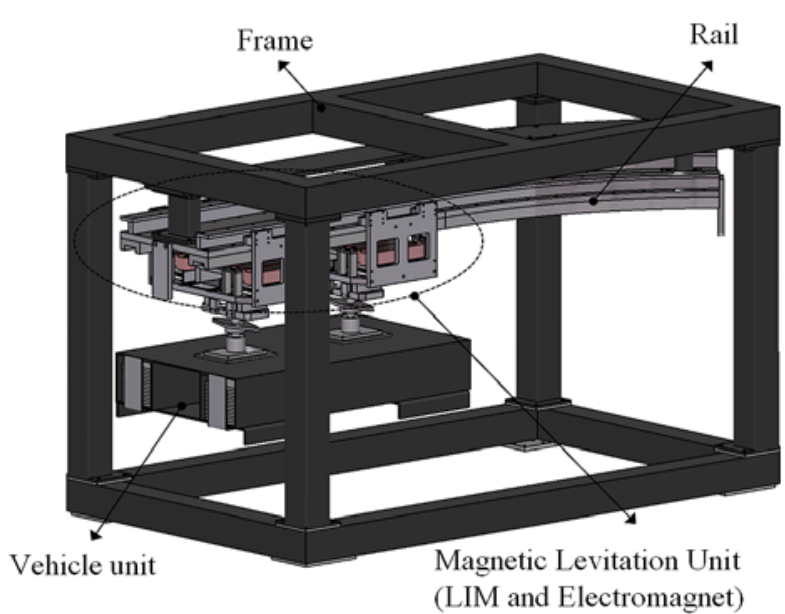

(a)

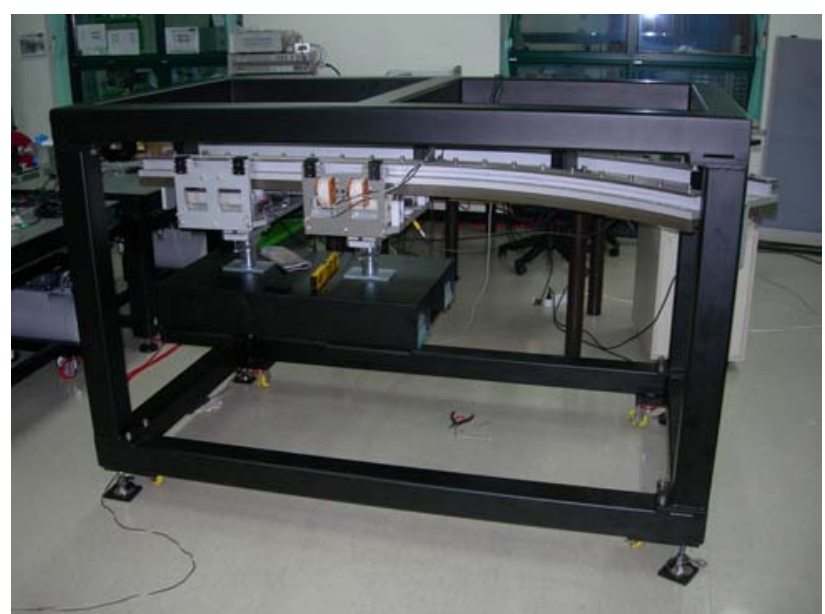

(b)

Response Surface Methodology (RSM) is generally used with two or three design variables, however we have seven design variables, so a mixed orthogonal array table was utilized. The reason for this is that a mixed orthogonal array table is an efficient way to study the effect of several design variables simultaneously with a number small of experiments and to plan matrix simulation trials. For each design variable combination the response value is determined by the $2 \mathrm{D}$ and $3 \mathrm{D}$ Finite Element Method (FEM). In this paper we use the reduced gradient algorithm, which can lead to the selection of the most desired set of variables. Figure 2 shows optimization procedure flow chart of RSM and kriging interpolation method. Figure 3 shows the application prototype model for applying the 
contactless delivery application. The response is determined and we evaluate the influence of each design variable on the objective function. Based on this method the weight of the optimized electromagnet could be reduced by $11.412 \%$ and normal force improved by $7.754 \%$, compared to the initially designed electromagnet.

Figure 2. Optimization flow chart.

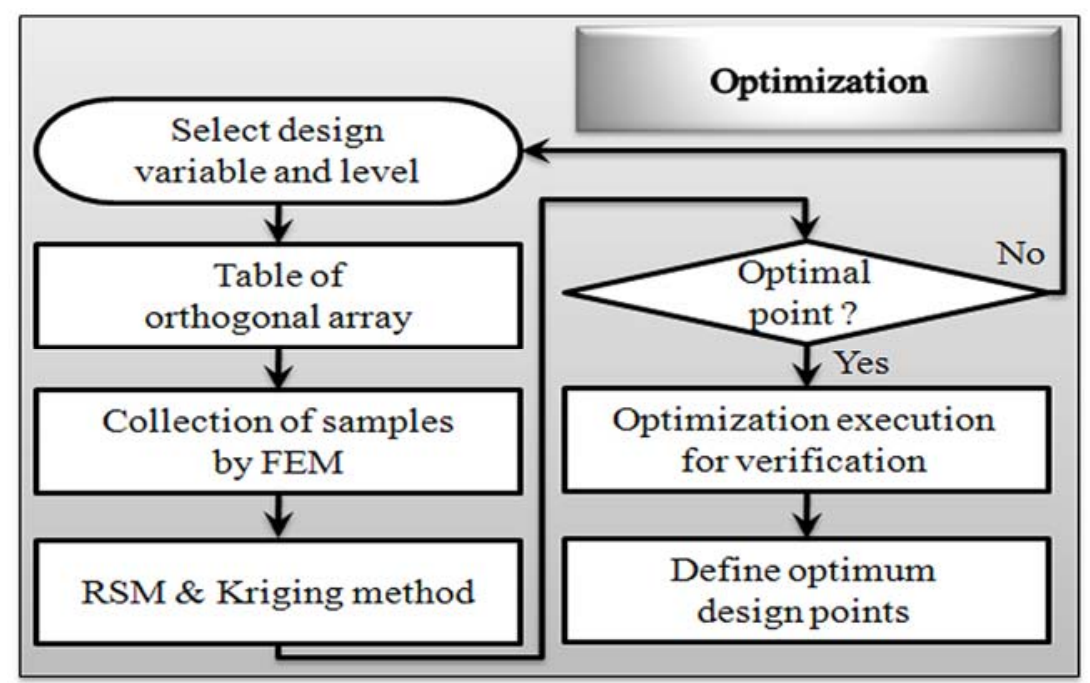

Figure 3. Application prototype.

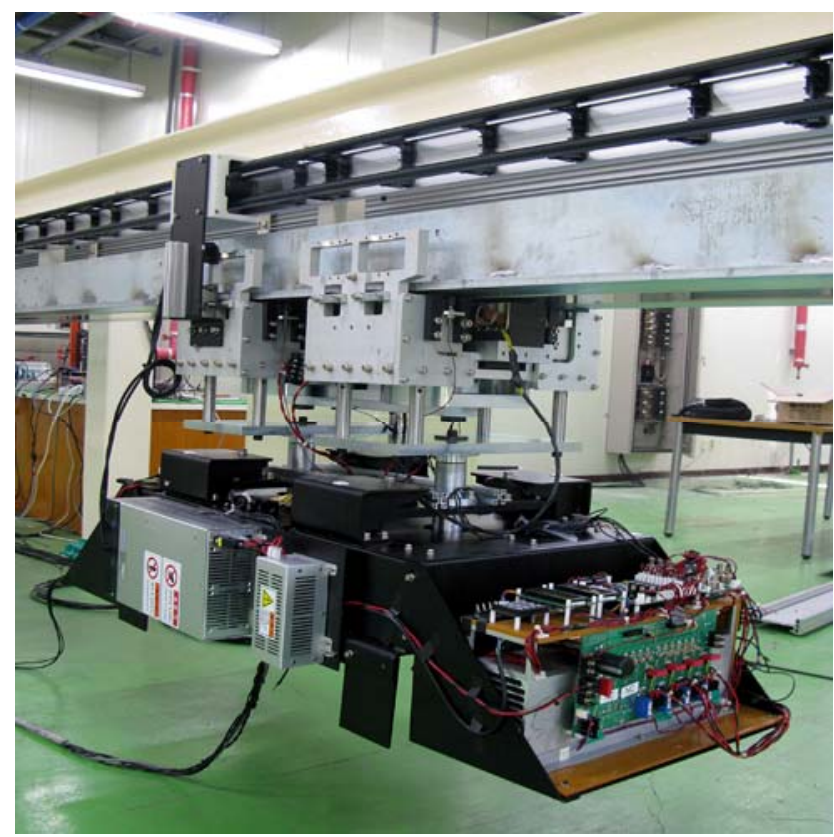

\section{Passive Guidance Control and Optimization of Electromagnet}

Let's assume that the guidance forces are generated by closed-loop control of levitation electromagnets. The lateral response of the electromagnet due to the airgap control is a force increasing with the lateral offset $\mathrm{X}$. This force is almost a linear function of the lateral offset of the electromagnets. Then we can imagine that the electromagnet will move laterally as a mass bound by a 
spring. As we know, a spring working on a mass is a mechanical resonant system, whose resonance frequency is given by:

$$
f_{\text {resonance }}=\frac{1}{2 \pi} \sqrt{\frac{\mathrm{K}_{\text {guide }}}{M}}
$$

where the $\mathrm{M}$ is the sum of the masses of the electromagnet and of part of the carried vehicle. We can easily imagine that any external action will cause a non-damped oscillating response of the lateral position. The value of $\mathrm{k}_{\text {guide }}$ can be designed to keep the disturbance from causing an excessive displacement. An example of the design problem is shown in Figure 4.

Figure 4. Lateral position model for electromagnets under constant levitation control: (a) passive guidance model; (b) simulation result for a $100 \mathrm{~N}$ disturbance.

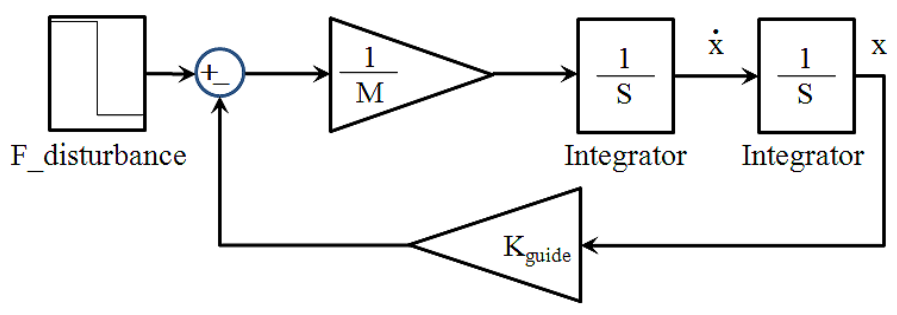

(a)

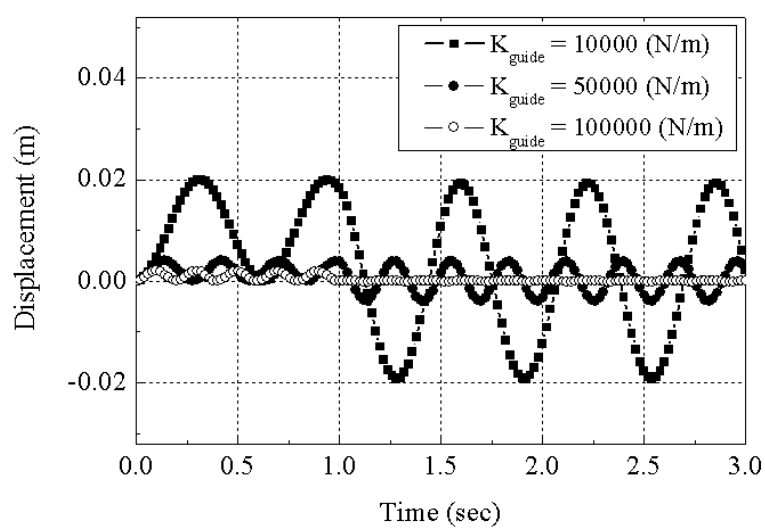

(b)

A total mass of $100 \mathrm{~kg}$ is assumed to be levitated with a constant gap. The sum of lateral position stiffness of the levitation magnets is set at $10,000 \mathrm{~N} / \mathrm{m}, 50,000 \mathrm{~N} / \mathrm{m}$ and $100,000 \mathrm{~N} / \mathrm{m}$, respectively. The lateral disturbance forces are assumed to be a constant value of $100 \mathrm{~N}$ for about 1 second. The simulation result shows that if the lateral position stiffness remains higher than $50,000 \mathrm{~N} / \mathrm{m}$, the lateral position deviation is smaller than $5 \mathrm{~mm}$ under constant $100 \mathrm{~N}$ disturbance.

In the experiment, a total mass of $200 \mathrm{~kg}$ including four levitation electromagnets is levitated under small deviation of electromagnet placement conditions, as shown in Figure 5. That is, a mass of $50 \mathrm{~kg}$ is levitated by one electromagnet. In the experiment, levitation magnets are controlled to maintain a constant gap length of $5 \mathrm{~mm}$. A position disturbance of $3.2 \mathrm{~mm}$ is applied at first and then removed. The lateral response of the experiment is shown in Figure 5(b). We can infer that if two electromagnets are positioned with small deviation from the original rail position, the guidance forces generated each electromagnet can be larger, but the resultant force becomes smaller because of the differential actuation scheme, so the position stiffness become smaller, the natural frequency becomes smaller and the lateral deviation becomes larger as the deviation of magnets becomes larger. Therefore, no-split positioning of electromagnets is better for lateral performance. 
Figure 5. Lateral position response of magnetically sprung mass under position disturbance: (a) experimental model; (b) lateral position response (experiment); (c) lateral position stiffness response (simulation); (d) lateral force response (simulation).

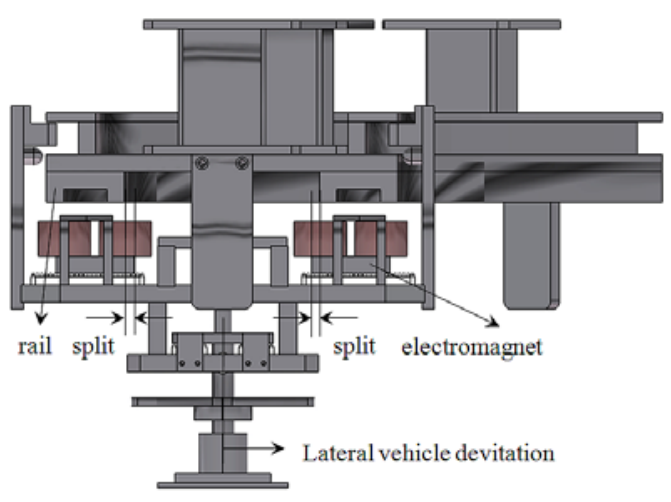

(a)

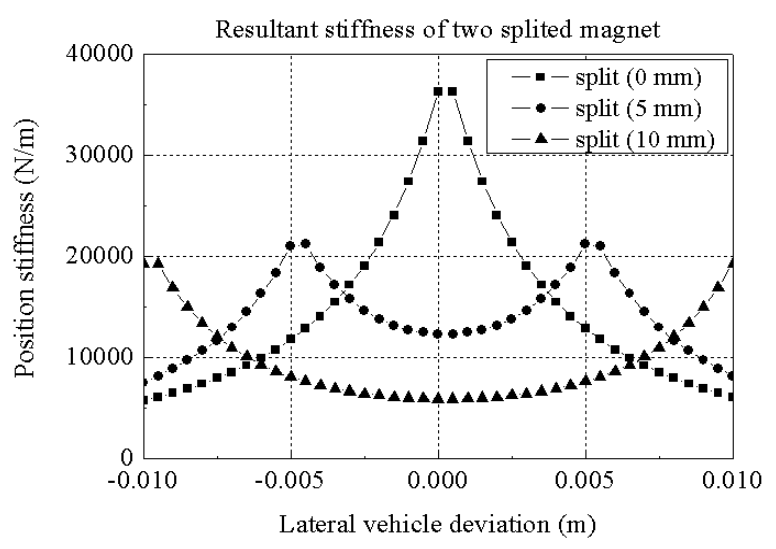

(c)

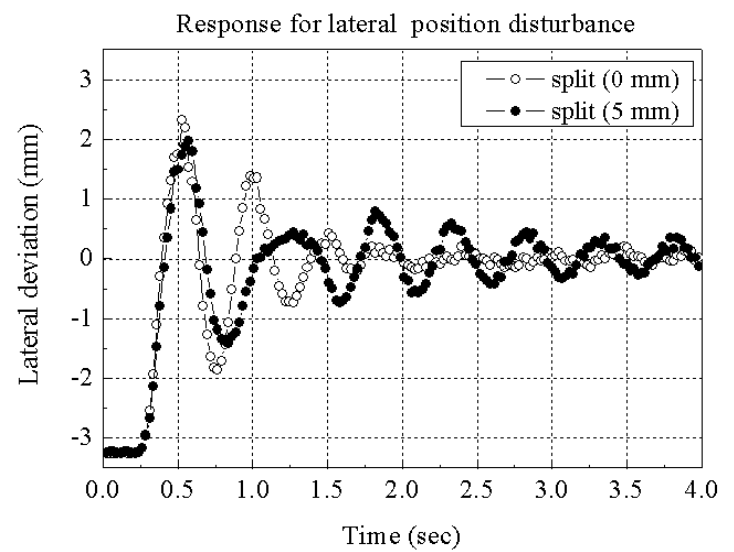

(b)

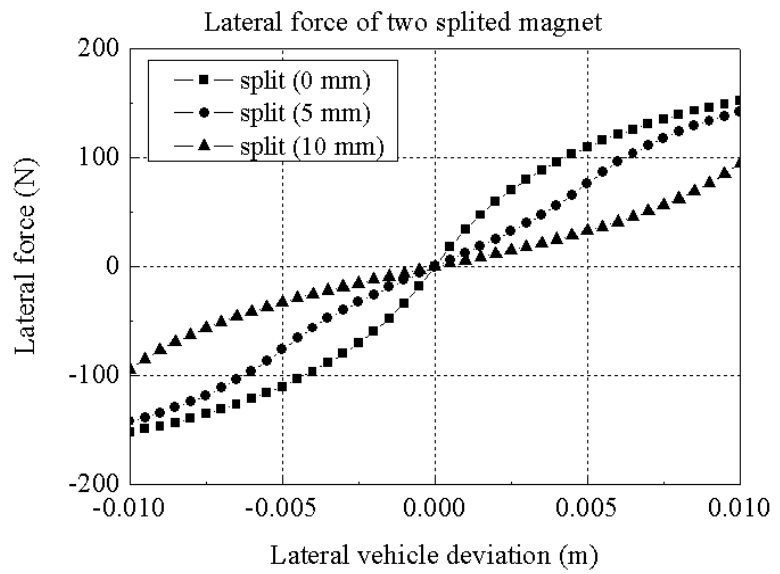

(d)

\section{Optimum Theory}

\subsection{Response Surface Methodology}

The RSM method can be readily adapted to develop an analytical model for a complex problem. With this analytical model, an objective function with constraints can be easily created and evaluated, and computation time can be saved. A polynomial approximation model is commonly used for a second-order fitted response $(u)$ and can be written as follows:

$$
\begin{gathered}
u=\beta_{0}+\sum_{j=l}^{k} \beta_{j} x_{j j}+\sum_{j=i}^{k} \beta_{j j} x_{j}^{2}+\sum_{j=l}^{k} \beta_{i j} x_{i} x_{j}+\varepsilon \\
\beta: \text { regression coefficients, } x: \text { design variables } \\
\varepsilon: \text { random error, } k: \text { number of design variables }
\end{gathered}
$$

The least squares method is used to estimate unknown coefficients. Matrix notations of the fitted coefficients and the fitted response model should be as shown in equation (3):

$$
\hat{\beta}=\left(X^{\prime} X\right)^{-1} X^{\prime} u, \hat{u}=X \hat{\beta}
$$


where $\hat{\beta}$ is a vector of the unknown coefficients which are usually estimated to minimize the sum of the squares of the error term. It should be evaluated at the data points. RSM can be applied in connection with FEM and the response actually represents FEM result.

\subsection{Kriging Interpolation Method}

Kriging is a method of interpolation named after a South African mining engineer D. G. Krige, who developed the technique while trying to increase accuracy in predicting ore reserves. In the kriging model, the global approximation model for a response $\mathrm{y}(\mathbf{x})$ is represented as:

$$
y(\mathbf{x})=\beta+v(\mathbf{x})
$$

where $\mathbf{x}$ is the design variable vector, $\beta$ is a constant, and $v(\mathbf{x})$ is the realization of a stochastic process. In Equation (4), $v(\mathbf{x})$ has the mean zero, variance $\sigma^{2}$, and non-zero covariance. The weight of electromagnet Weight $t_{\text {total }}$ is replaced by $y(\mathbf{x})$ to make a surrogate approximation model. Let $y(\mathbf{x})$ be an approximation model. Hereafter, $\wedge$ means the estimator. When the mean squared error between $y(\mathbf{x})$ and $\hat{y(\mathbf{x})}$ is minimized, $\hat{y(\mathbf{x})}$ becomes:

$$
\hat{y(\mathbf{x})}=\hat{\beta}+\mathbf{r}^{T}(\mathbf{x}) \mathbf{R}^{-1}(\mathbf{y}-\hat{\beta} \mathbf{q})
$$

where $\mathbf{r}$ is the correlation vector, $\mathbf{R}$ is the correlation matrix, $\mathbf{y}$ is the observed data and $\mathbf{q}$ is the unit vector. The definitions of $\mathbf{R}$ and $\mathbf{r}$ are well explained in Refs [8,9].

The unknown correlation parameters of $\theta_{1}, \theta_{2}, \ldots, \theta_{n}$ defined in $\mathbf{R}$ are calculated from the formulation according to:

$$
\text { maximize } \quad-\frac{\left[n_{s} \ln \left(\hat{\sigma^{2}}\right)+\ln |\mathbf{R}|\right]}{2}
$$

where $\theta_{i}(\mathrm{i}=1,2, \ldots, \mathrm{n})>0$. In this study, a GRG (generalized reduced gradient) algorithm built in the Excel program was utilized to determine the optimum parameters.

\section{Optimum Design}

\subsection{Metamodel and Design Variable}

Optimization formulation:

$$
\begin{gathered}
\text { Minimize: Weight } t_{\text {total }}\left(d v_{1}, \ldots, d v_{7}\right) \\
\text { Subject to } \quad F_{\text {normal }}\left(d v_{1}, \ldots, d v_{7}\right) \geq 611.689
\end{gathered}
$$

Figure 6 shows the prototype electromagnet of the reference model which is analyzed. The comparison of the static force obtained from FEM simulation and experimental test is shown in Figure 7. From the results, the use of FEM is validated, as it can be observed, since the comparison of normal force by simulation and experiment test shown in Figure 7 displays good agreement. Selection of the design variables is very important setup in optimization procedure. 
Figure 6. Prototype of the electromagnet.

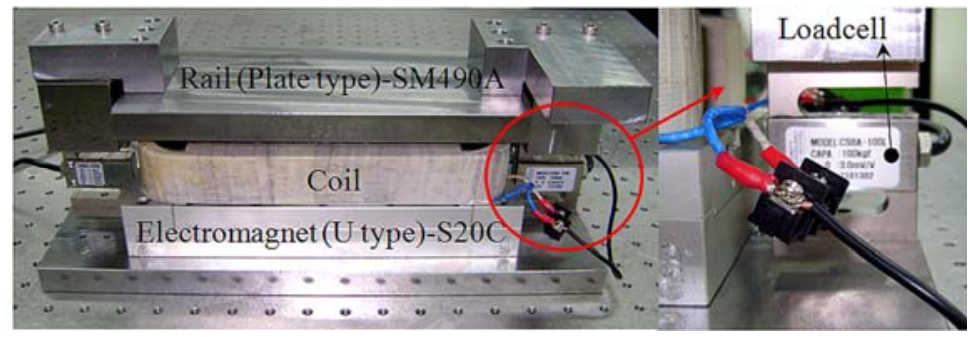

Figure 7. Comparison of FEM and experiment (reference model).

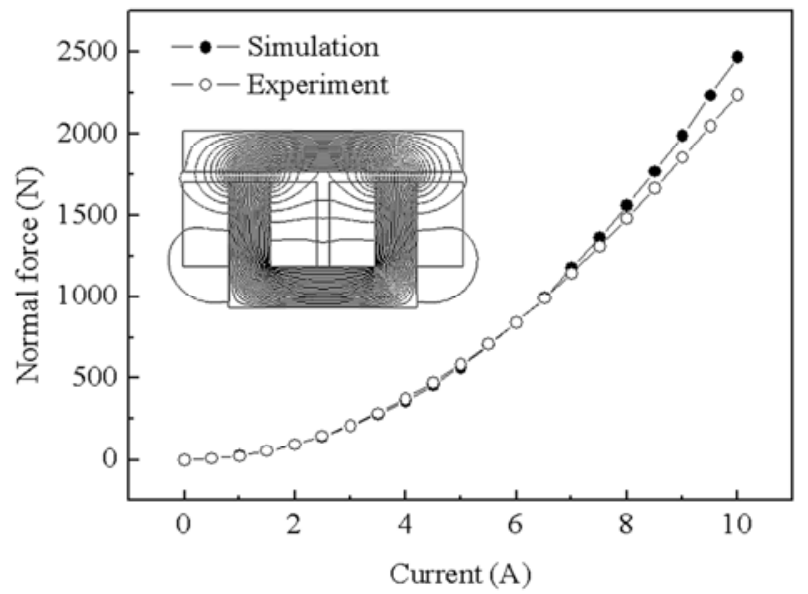

Seven dimensions are selected as the design variables as shown in Figure 8, which shows the flux density vector in electromagnet of 2D and 3D FEM. The magnetization characteristics of the S20C, SM490A material is as shown in Figure 9. Table 1 shows the design variables and levels.

Table 1. Design variable and level.

\begin{tabular}{cccccccc}
\hline Design variable Level & $d v_{1}$ & $d v_{2}$ & $d v_{3}$ & $d v_{4}$ & $d v_{5}$ & $d v_{6}$ & $d v_{7}$ \\
\hline-1 & 16 & 45 & 16 & 40 & 7 & 16 & 144 \\
0 & 20 & 50 & 20 & 45 & 11 & 20 & 180 \\
1 & 24 & 55 & 24 & 50 & 15 & 24 & 216 \\
\hline
\end{tabular}

Figure 8. Design variables and flux pattern (2D and 3D FEM).

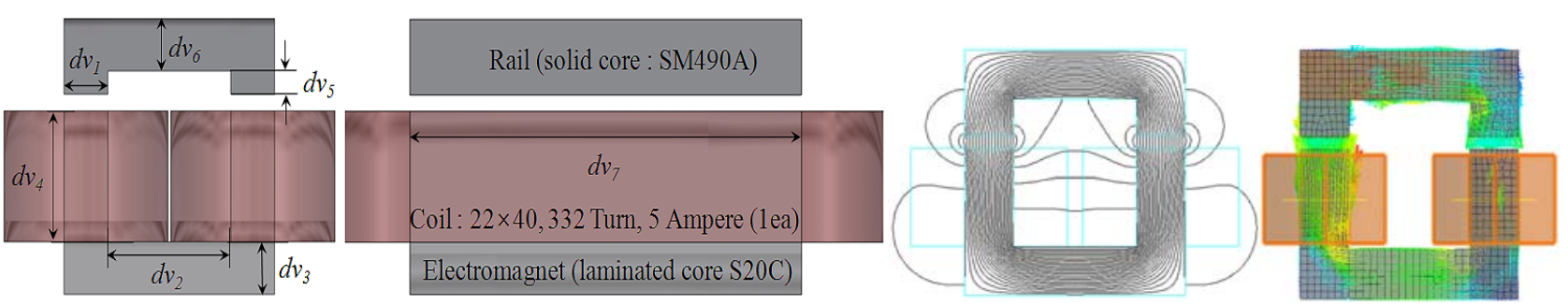


Figure 9. Magnetization curve of the core material used.

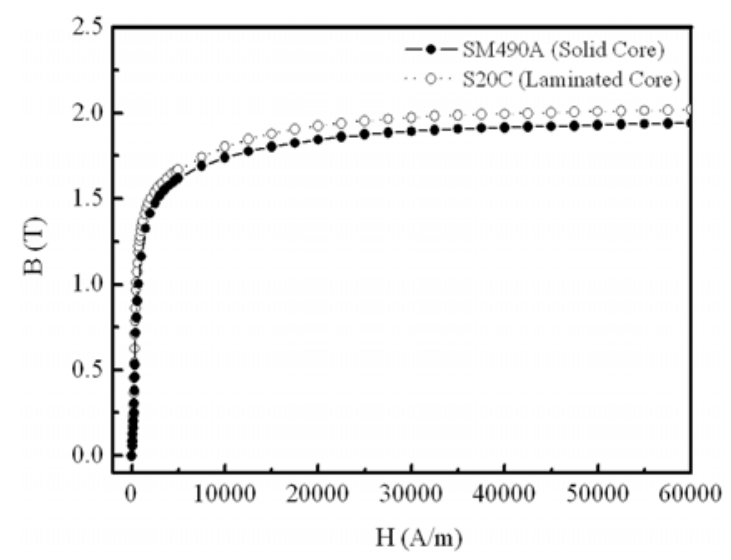

\subsection{Response Surface Methodology}

Table 2 shows the table of mixed orthogonal array and simulation results by 2D FEM. The mixed orthogonal array table is determined by considering the number of the design variables and each level of them. After getting experimental data by FEM, the function to draw a response surface is extracted. In order to determine equations of the response surface for each response value (weight, normal force), several experimental designs have been developed to establish the approximate equation using the smallest number of experiments. The purpose of this paper is to minimize the objective function (Weight $\left.t_{\text {total }}\right)$ with constraints of normal force $\left(F_{\text {normal }}\right)$. The two fitted second order polynomial of objective functions for the seven design variables are as follows:

$$
\begin{aligned}
F_{\text {normal }}= & 148.75+26.1 d v_{1}-2.41 d v_{2}-10.02 d v_{3}-32.18 d v_{4}-1.63 d v_{5}+35.16 d v_{6} \\
+ & 3.01 d v_{7}-7.14 E-2 d v_{1}^{2}+1.3 E-3 d v_{2}^{2}+0.23 d v_{3}^{2}+0.35 d v_{4}^{2}+5.56 E-2 d v_{5}^{2} \\
- & 0.9 d v_{6}^{2}-3.72 E-4 d v_{7}^{2} \\
\text { Weight }_{\text {total }}= & 8.765+0.212 d v_{1}-0.248 d v_{2}-0.154 d v_{3}-0.488 d v_{4}-0.113 d v_{5}+0.264 d v_{6} \\
& +0.065 d v_{7}+1.84 E-3 d v_{1}^{2}+2.8 E-3 d v_{2}^{2}+6.54 E-3 d v_{3}^{2}+5.95 E-3 d v_{4}^{2} \\
& +7.58 E-3 d v_{5}^{2}-4.05 E-3 d v_{6}^{2}+2.64 E-6 d v_{7}^{2}
\end{aligned}
$$

The adjusted coefficients of the multiple determination $\mathrm{R}_{\text {adj }}^{2}$ for normal force and weight are weight $_{\text {total }}(99 \%)$ and $F_{\text {normal }}(100 \%)$. Normal force and weight of experiment result according to change of the design variables are shown in Table 2. As many parameters are defined as design variables, a large simulation time is required due to the large number of required experiments. Therefore, it is necessary to establish the significant parameters to investigate the influence on the design result. The Pareto chart of normal force and weight shows the magnitude and importance of an effect. This chart displays the absolute value of the effects. The geometries of electromagnet can be defined by seven parameters, as shown in Table 1 and Figure 8 . The most effective design variables of normal force and weight are $d v_{7}, d v_{1}$ according to the Pareto chart in Figure 10. 
Table 2. Table of mixed orthogonal array $L_{18}\left(2^{1} \times 3^{7}\right)$.

\begin{tabular}{|c|c|c|c|c|c|c|c|c|c|}
\hline Exp. & $\boldsymbol{d} \boldsymbol{v}_{\boldsymbol{1}}$ & $\boldsymbol{d} \boldsymbol{v}_{\mathbf{2}}$ & $\boldsymbol{d}_{\boldsymbol{3}}$ & $\boldsymbol{d} \boldsymbol{v}_{\boldsymbol{4}}$ & $\boldsymbol{d} \boldsymbol{v}_{\mathbf{5}}$ & $\boldsymbol{d} \boldsymbol{v}_{\boldsymbol{6}}$ & $\boldsymbol{d v}_{\boldsymbol{7}}$ & Normal force (N) & Weight (kg) \\
\hline 1 & 16 & 45 & 16 & 40 & 7 & 16 & 144 & 382.10 & 8.706 \\
2 & 16 & 50 & 20 & 45 & 11 & 20 & 180 & 473.67 & 12.153 \\
3 & 16 & 55 & 24 & 50 & 15 & 24 & 216 & 564.15 & 16.366 \\
4 & 20 & 45 & 16 & 45 & 11 & 24 & 216 & 682.56 & 15.433 \\
5 & 20 & 50 & 20 & 50 & 15 & 16 & 144 & 450.22 & 10.979 \\
6 & 20 & 55 & 24 & 40 & 7 & 20 & 180 & 570.56 & 13.699 \\
7 & 24 & 45 & 20 & 40 & 15 & 20 & 216 & 795.40 & 16.803 \\
8 & 24 & 50 & 24 & 45 & 7 & 24 & 144 & 531.60 & 12.66 \\
9 & 24 & 55 & 16 & 50 & 11 & 16 & 180 & 651.24 & 14.064 \\
10 & 16 & 45 & 24 & 50 & 11 & 20 & 144 & 379.41 & 10.277 \\
11 & 16 & 50 & 16 & 40 & 15 & 24 & 180 & 473.76 & 12.094 \\
12 & 16 & 55 & 20 & 45 & 7 & 16 & 216 & 568.30 & 13.874 \\
13 & 20 & 45 & 20 & 50 & 7 & 24 & 180 & 570.38 & 13.637 \\
14 & 20 & 50 & 24 & 40 & 11 & 16 & 216 & 682.15 & 15.453 \\
15 & 20 & 55 & 16 & 45 & 15 & 20 & 144 & 450.16 & 10.946 \\
16 & 24 & 45 & 24 & 45 & 15 & 16 & 180 & 659.92 & 14.604 \\
17 & 24 & 50 & 16 & 50 & 7 & 20 & 216 & 789.93 & 16.646 \\
18 & 24 & 55 & 20 & 40 & 11 & 24 & 144 & 528.87 & 12.400 \\
\hline
\end{tabular}

Figure 10. Pareto chart of the standardized effects (alpha=0.05) (a) Normal force response (b) Total weight response.

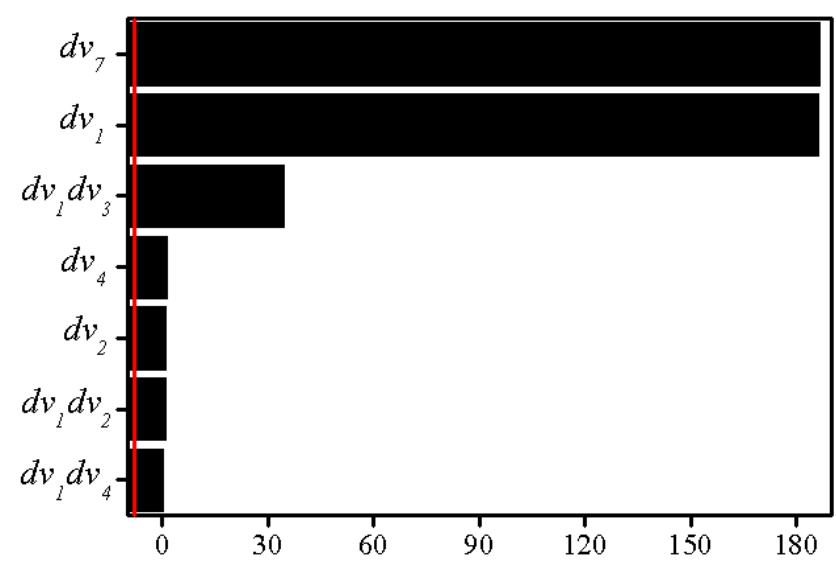

(a)

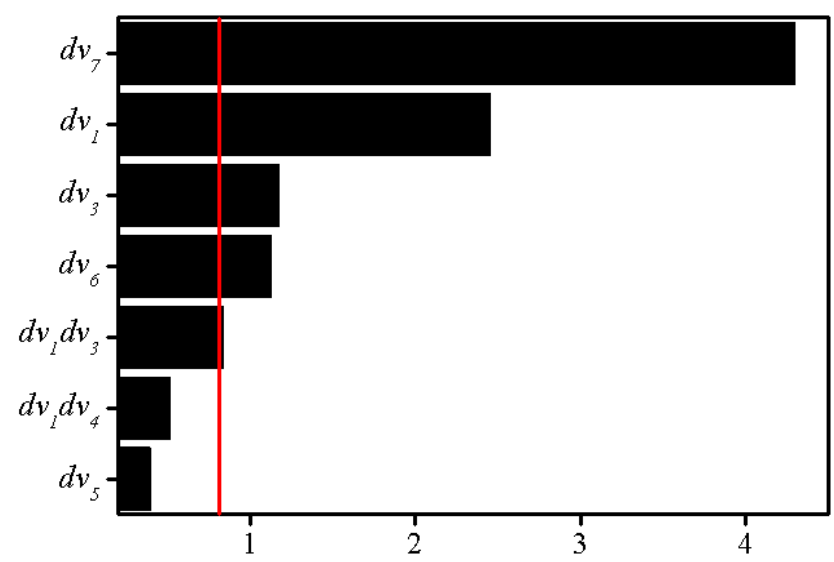

(b)

The reference line corresponds to alpha $=0.05 ; 95 \%$ confidence interval.

The values of ineffective design variables are determined by RSM. Figure 11 shows response optimization to find optimal solution according to response curves. The slope of the response function in Figure 11 shows the sensitivity of design variable. According to the response optimization the most sensitive design variables of normal force and weight are $d v_{7}, d v_{1}$. 
Figure 11. Response optimization.

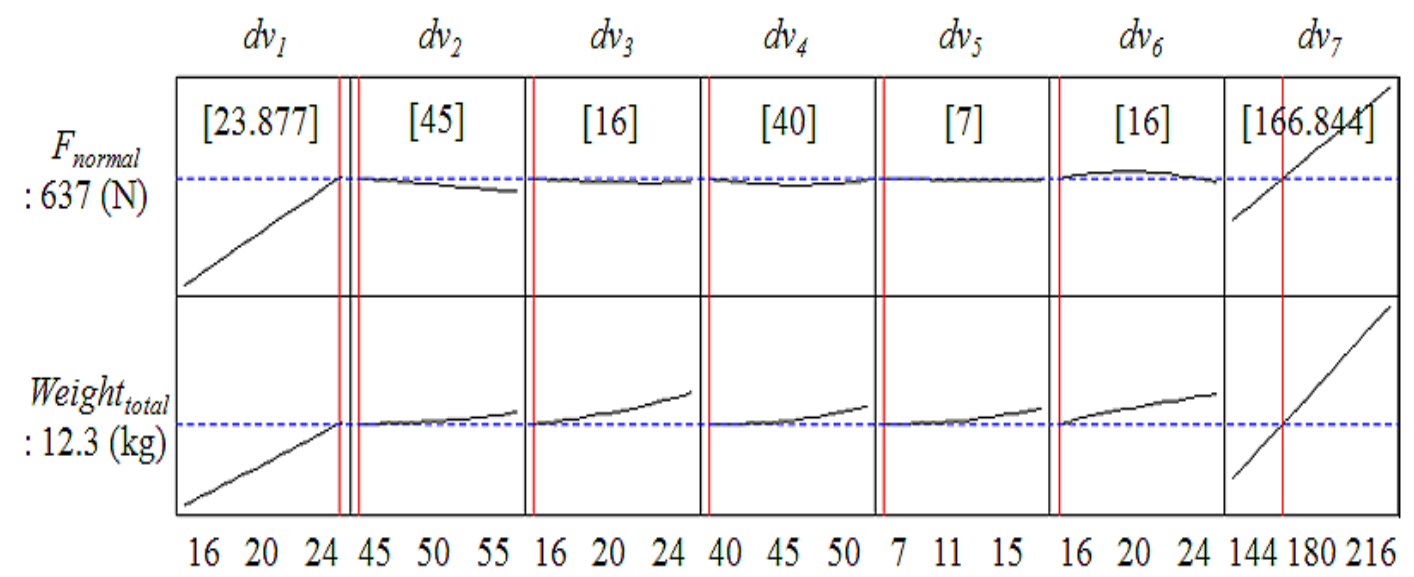

\subsection{Kriging Interpolation Method}

The purpose of this paper is to minimize the objective function (Weight $t_{\text {total }}$ ) with the constraint of normal force $\left(F_{\text {normal }}\right)$. The kriging interpolation method, the optimum parameters of $\theta_{1}, \ldots, \theta_{7}$ are determined by solving Equation (6). Then, the estimator $\beta$ is calculated. In Tables 3 and 4, the optimal point is searched to find the point of less than $11.412 \%$ of the weight and greater than $7.754 \%$ of the normal force of the initially designed electromagnet in the magnetic levitation system. The optimum estimators are shown as Table 4. The optimum solution is the same in the RSM and kriging interpolation method. The reason is that the optimum solution is near the boundary value. However, response values, Weight $t_{\text {total }}$ and $F_{\text {normal }}$ by RSM and kriging method, respectively, are different. The simulation result of the predicted optimum set is shown Table 5, displaying good agreement.

Table 3. Optimum level and size.

\begin{tabular}{lccccccc} 
& \multicolumn{8}{c}{ Design variable } \\
\hline \multicolumn{1}{c}{ Model } & $\boldsymbol{d} \boldsymbol{v}_{\boldsymbol{1}}$ & $\boldsymbol{d} \boldsymbol{v}_{2}$ & $\boldsymbol{d} \boldsymbol{v}_{3}$ & $\boldsymbol{d} \boldsymbol{v}_{4}$ & $\boldsymbol{d} \boldsymbol{v}_{5}$ & $\boldsymbol{d} \boldsymbol{v}_{\boldsymbol{6}}$ & $\boldsymbol{d} \boldsymbol{v}_{7}$ \\
\hline Initial & 20 & 50 & 20 & 40 & 15 & 20 & 180 \\
Optimum (RSM) & 23.877 & 45 & 16 & 40 & 7 & 16 & 166.844 \\
Optimum (Kriging) & 23.877 & 45 & 16 & 40 & 7 & 16 & 166.844 \\
\hline
\end{tabular}

Table 4. Optimum $\beta$ and correlation parameters for kriging models $\left(\mathrm{N}_{\mathrm{s}}=100\right)$.

\begin{tabular}{ccccccccc}
\hline \multirow{2}{*}{ Response } & \multicolumn{7}{c}{ Correlation parameter (corresponding design variable) } \\
\cline { 2 - 9 } & $\boldsymbol{\theta}_{\boldsymbol{I}}\left(\boldsymbol{d} \boldsymbol{v}_{\boldsymbol{1}}\right)$ & $\boldsymbol{\theta}_{\mathbf{2}}\left(\boldsymbol{d} \boldsymbol{v}_{\boldsymbol{2}}\right)$ & $\boldsymbol{\theta}_{\mathbf{3}}\left(\boldsymbol{d} \boldsymbol{v}_{\mathbf{3}}\right)$ & $\boldsymbol{\theta}_{\boldsymbol{4}}\left(\boldsymbol{d} \boldsymbol{v}_{4}\right)$ & $\boldsymbol{\theta}_{\boldsymbol{5}}\left(\boldsymbol{d} \boldsymbol{v}_{5}\right)$ & $\boldsymbol{\theta}_{\boldsymbol{6}}\left(\boldsymbol{d} \boldsymbol{v}_{\boldsymbol{6}}\right)$ & $\boldsymbol{\theta}_{7}\left(\boldsymbol{d} \boldsymbol{v}_{7}\right)$ & $\boldsymbol{\beta}$ \\
\hline Weight $t_{\text {total }}$ & $3.026 \mathrm{e}-3$ & $2.304 \mathrm{e}-4$ & $0.909 \mathrm{e}-3$ & $0.201 \mathrm{e}-3$ & $6.318 \mathrm{e}-5$ & $1.195 \mathrm{e}-3$ & $1.289 \mathrm{e}-2$ & 16.0023 \\
$F_{\text {normal }}$ & $1.319 \mathrm{e}-2$ & $1.382 \mathrm{e}-5$ & $3.437 \mathrm{e}-6$ & $1.172 \mathrm{e}-5$ & $7.819 \mathrm{e}-6$ & $2.320 \mathrm{e}-6$ & $4.087 \mathrm{e}-2$ & 587.009 \\
\hline
\end{tabular}


Table 5. Comparison of initial and optimum model.

\begin{tabular}{clcc}
\hline \multicolumn{1}{c}{ Model } & Weight (kg) & Normal force (N) \\
\hline \multirow{2}{*}{ Initial } & 2D FEM & 13.319 & 578.5 \\
& 3D FEM & 13.319 & 573.48 \\
& Error(2D vs. 3D) \% & 0 & 0.86 \\
Optimum & RSM (predicted) & 12.300 & 637 \\
& Kriging method (predicted) & 12.107 & 611.68 \\
& FEM (verification) & 11.799 & 611.70 \\
& Error (RSM vs. FEM) \% & -4.073 & -3.972 \\
& Error (Kriging vs. FEM) \% & -2.610 & 0.003 \\
Variation between initial and optimum FEM \% & -11.412 & 7.754 \\
\hline
\end{tabular}

\section{Conclusions}

This paper deals with optimum design of a lightweight levitation electromagnet on a vehicle, which also provides passive guide force, in a magnetic levitation system for contactless delivery applications. The optimum design procedure is introduced to design of electromagnet in the magnetic levitation system to reduce its weight and to improve the normal force of the initially designed electromagnet in the magnetic levitation system using several design variables. The most effective design variables are extracted by Pareto chart. The most desired set is determined by RSM and the kriging interpolation method and the influence of each design variables on the objective function can be obtained. This can efficiently increase the precision of the optimization and reduce the number of experiments in the optimization design using the proposed methodologies.

\section{References}

1. D'Arrigo, A.; Rufer, A. Integrated electromagnetic levitation and guidance system for the swissmetro project. In Proceedings of International Conference on Magnetically Levitated Systems and Linear Drives (MAGLEV' 2000), Rio de Janeiro, Brazil, 7-10 June 2000; pp. 263-268.

2. Tzeng, Y.K.; Wang, T.C. Optimal design of the electromagnetic levitation with permanent and electro magnets. IEEE Trans. Magn. 1994, 30, 4731-4733.

3. Kim, Y.J.; Shin, P.S.; Kang, D.H.; Cho, Y.H. Design and analysis of electromagnetic system in a magnetically levitated vehicle, KOMAG-01. IEEE Trans. Magn. 1992, 28, 3321-3323.

4. Onuki, T.; Tota, Y. Optimal design of hybrid magnet in Maglev system with both permanent and electromagnets. IEEE Trans. Magn. 1993, 29, 1783-1786.

5. Hong, D.K.; Woo, B.C.; Chang, J.H.; Kang, D.H. Optimum design of TFLM with constraints for weight reduction using characteristic function. IEEE Trans. Magn. 2007, 43, 1613-1616.

6. Hong, D.K.; Choi, S.C.; Ahn, C.W. Robust optimization design of overhead crane with constraint using the characteristic functions. Int. J. Precision Eng. Manuf. 2006, 7, 12-17.

7. Kim, J.M.; Lee, S.H.; Choi, Y.K. Decentralized $\mathrm{H}_{\infty}$ control of Maglev systems. In Proceedings of IEEE Industrial Electronics, IEEE IECON 2006, Paris, France, 6-10 November 2006; pp. 418-423. 
8. Lee, K.H.; Kang, D.H. Structural optimization of an automotive door using the kriging interpolation method. Proc. Inst. Mech. Eng. D J. Automobile Eng. 2007, 221, 1525-1534.

9. Guinta, A.; Watson, L. A comparison of approximation modeling techniques polynomial versus interpolating models. In Proceedings of the 7thAIAA/USAF/NASA/ISSMO Symposium on Multi-disciplinary Analysis and Optimization, Saint Louis, MO, USA, September 1998; pp. 392-440.

(C) 2010 by the authors; licensee MDPI, Basel, Switzerland. This article is an Open Access article distributed under the terms and conditions of the Creative Commons Attribution license (http://creativecommons.org/licenses/by/3.0/). 